\title{
Presynaptic Remodeling Contributes to Activity-Dependent Synaptogenesis
}

\author{
Irina Nikonenko, Pascal Jourdain, and Dominique Muller \\ Department of Pharmacology, University Medical Center, 1211 Geneva 4, Switzerland
}

\begin{abstract}
Induction of long-term potentiation and application of short periods of anoxia/hypoglycemia result in the growth of dendritic filopodia and formation of new spines. Here we investigated whether these conditions also affected the morphology of presynaptic structures. Using confocal imaging of DiI-labeled axons, electron microscopy, and stereological analyses, we show that short anoxia/hypoglycemia and theta burst stimulation induced rapid, calcium-dependent growth of presynaptic filopodia-like protrusions and remodeling of presynaptic varicosities. Three-dimensional reconstruction of axonal outgrowths revealed that, within $30 \mathrm{~min}$, they made contacts and triggered the formation of a postsynaptic density on the target cell. Interestingly, these axonal filopodia first established synapses with the dendritic shaft and later mostly with spines. They also contributed to the formation of multi-innervated spines. Because these presynaptic growth mechanisms depended on NMDA receptor activation, we investigated whether a diffusing messenger could be involved. We found that blockade of nitric oxide synthase prevented these changes, and conversely, a nitric oxide donor could reproduce them. A model is presented that proposes that activation of NMDA receptors and subsequent release of nitric oxide could trigger the growth of presynaptic filopodia, which, in turn, play an active role in synaptogenesis and spine formation.
\end{abstract}

Key words: anoxia/hypoglycemia; LTP; presynaptic morphology; nitric oxide; rat; hippocampus

\section{Introduction}

Excitatory synapses on dendritic spines exhibit a high degree of structural plasticity (Luscher et al., 2000). Spines are characterized by dynamic movements triggered by actin-based mechanisms (Fischer et al., 1998; Star et al., 2002) and may change shape (Korkotian and Segal, 1999, 2001; Segal et al., 2000), exhibit remodeling of their postsynaptic density (PSD) (Geinisman, 1993; Buchs and Muller, 1996; Marrs et al., 2001; Toni et al., 2001), or be formed de novo (Engert and Bonhoeffer, 1999; Goldin et al., 2001; Jourdain et al., 2002) under the influence of activity and calcium. Recent studies showed that induction of long-term potentiation triggers the formation of dendritic filopodia (MaleticSavatic et al., 1999), considered precursors of spines (Dailey and Smith, 1996; Ziv and Smith, 1996; Fiala et al., 1998), as well as a direct formation of new spines or new types of synapses (Engert and Bonhoeffer, 1999; Toni et al., 1999). Remodeling of postsynaptic structures has thus been proposed to play a major role in activity-dependent synaptogenesis. Other studies, however, also provided evidence for presynaptic morphological changes; stimulation of developing neuronal cultures results in actindependent formation and growth of axonal filopodia (Hatada et al., 2000; Chang and De Camilli, 2001; Colicos et al., 2002; Tashiro et al., 2003) as well as formation of functional presynaptic boutons (Ma et al., 1999; De Paola et al., 2003). The role of these presynaptic changes in synaptogenesis, however, remains

Received March 31, 2003; revised July 8, 2003; accepted July 21, 2003.

This work was supported by the Swiss National Science Foundation, the SCOPES program, the 0tt foundation, the De Reuter Foundation, and the Novartis Foundation. We thank M. Moosmayer and L. Parisi for excellent technical support and Fred Pillonel for photographic work.

Correspondence should be addressed to Dominique Muller at the above address. E-mail: dominique.muller@medecine.unige.ch.

Copyright $\odot 2003$ Society for Neuroscience $\quad 0270-6474 / 03 / 238498-08 \$ 15.00 / 0$ unclear. It is not known whether these mechanisms are purely developmental or whether they can also be triggered as a part of a plasticity program under more physiological conditions. Furthermore, there is no direct evidence that these presynaptic filopodia participate in the formation of mature synapses, and little is known about the mechanisms that affect their growth.

Here we investigated some of these issues using organotypic hippocampal slice cultures as a model and tested whether anoxia/ hypoglycemia and theta burst stimulation, two conditions that were shown to induce morphological plasticity, also triggered these processes. Our results suggest that activation of postsynaptic NMDA receptors and subsequent release of an intercellular messenger, nitric oxide, can stimulate a presynaptic plasticity, which in turn contributes to synaptogenesis.

\section{Materials and Methods}

Organotypic slice cultures. Hippocampal organotypic slice cultures were prepared from 7-d-old rats and maintained $10-15 \mathrm{~d}$ in culture as described (Stoppini et al., 1991). Only slice cultures satisfying criteria of good quality and preservation (general appearance, absence of swollen soma, dendrites, or varicosities, and size of evoked responses) were included in the study. We were also careful to use the same batches of slices cultures for test and control experiments (i.e., slice cultures prepared on the same day from littermates). All electrophysiology and confocal imaging experiments were performed with slice cultures placed on an infrapatch setup (Luigs and Neuman) under continuous perfusion $(4 \mathrm{ml} /$ min) with a medium containing (in $\mathrm{mm}$ ): $124 \mathrm{NaCl}, 1.6 \mathrm{KCl}, 2.5 \mathrm{CaCl}_{2}$, $1.5 \mathrm{MgCl}_{2}, 24 \mathrm{NaHCO}_{3}, 1.2 \mathrm{KH}_{2} \mathrm{PO}_{4}, 10$ glucose, and 2 ascorbic acid, $\mathrm{pH}$ 7.4, temperature $33^{\circ} \mathrm{C}$. EPSPs were evoked by stimulation of a group of Schaffer collaterals and recorded in CA1 pyramidal neurons. Anoxia/ hypoglycemia was produced by replacing oxygen with $\mathrm{N}_{2}$ and switching to a medium containing sucrose $(10 \mathrm{~mm})$ instead of glucose for $2 \mathrm{~min}$ (Jourdain et al., 2002). Theta burst stimulation consisted of five trains at $5 \mathrm{~Hz}$ each composed of four pulses at $100 \mathrm{~Hz}$, repeated three times at 10 

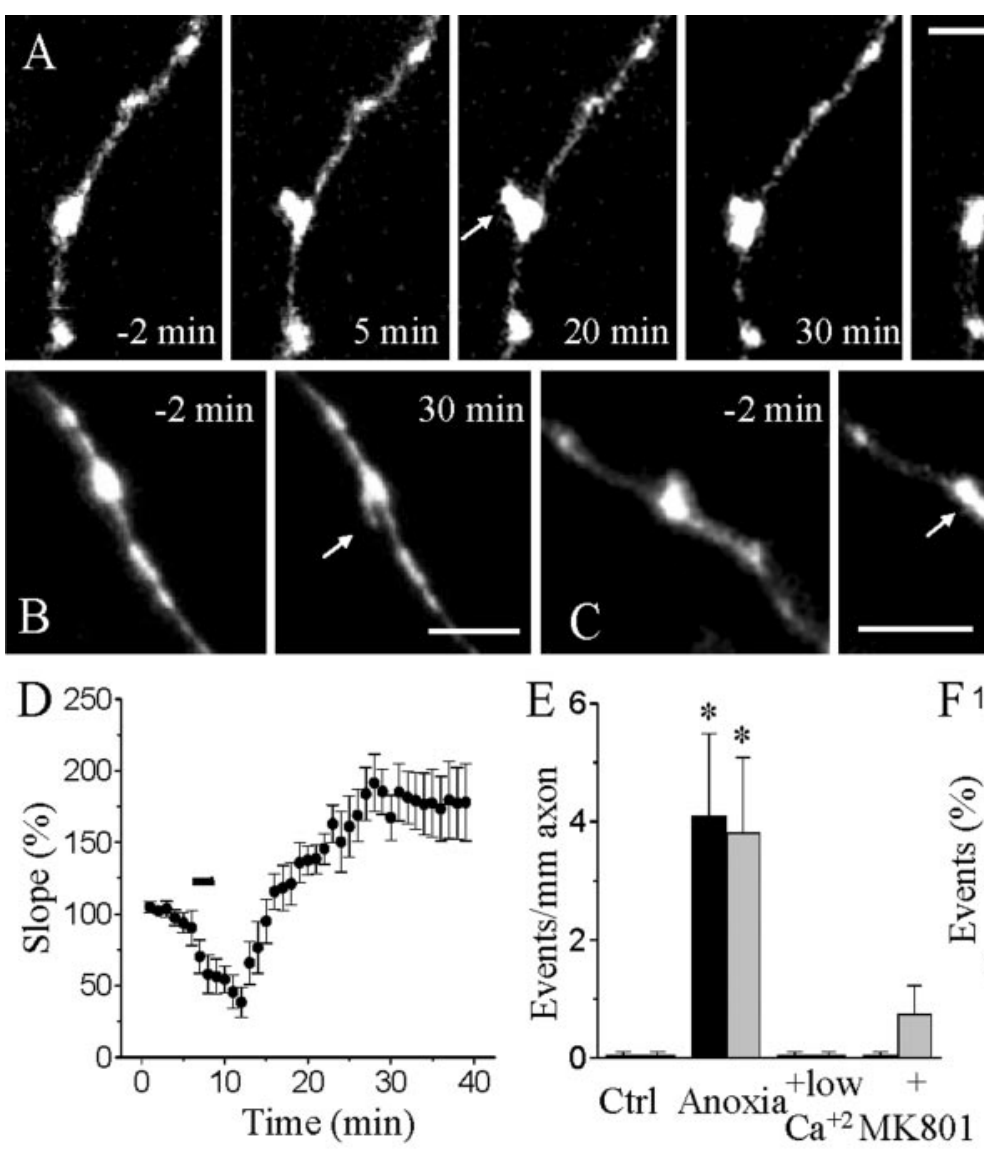

Figure 1. Confocal time-lapse imaging of presynaptic structural changes induced by short (2 min) anoxia/hypoglycemia in a hippocampal organotypic slice culture. $A$, Time sequence analysis illustrating the growth and retraction of a protrusion (arrow) emerging from a Dil-labeled presynaptic varicosity. $B$, Another example of presynaptic axonal outgrowth (arrow). C, Case of varicosity remodeling. Scale bars, $5 \mu \mathrm{m}$. D, Lasting enhancement of the slope of evoked EPSCs induced by application of short (2 $\mathrm{min}$ ) anoxia/hypoglycemia. Data are mean \pm SEM of five experiments. E, Statistical analysis of the number of cases of axonal outgrowths (black columns) and varicosity remodeling (gray columns) detected under the indicated conditions $[n=16$ in control (Ctrl) conditions, without stimulation; $n=17$ in anoxia; $n=12$ in anoxia + low calcium; $n=16$ in anoxia + MK801; $p<0.05$, $t$ test). F, Time of onset of axonal outgrowths and varicosity remodeling images after anoxia/hypoglycemia.

sec intervals. The same protocols were applied for the electron microscopy (EM) studies, except that slice cultures were kept in an interface chamber.

Confocal imaging. Axons of CA3 pyramidal neurons were labeled with DiI according to standard methods (Kirov and Harris, 1999). Briefly, fine crystals of DiI were delivered by the tip of a glass electrode to the middle part of the CA1 stratum radiatum to obtain retrograde labeling of CA3 neurons and Schaffer collaterals. A few tens of axons were labeled per slice culture, but only one or two labeled axonal segments were analyzed per slice. The analysis was performed at a distance of $\sim 200 \mu \mathrm{m}$ from the injection site in the external third of the stratum radiatum adjacent to the CA2 area, whereas the cell soma of labeled neurons was in the CA3 area at $\sim 300-400 \mu \mathrm{m}$ from the site of analysis. Imaging was performed using an argon laser (488 nm) and a Bio-Rad (Hercules, CA) MRC 1024 confocal microscope (Jourdain et al., 2002). Images of axonal segments of 60-140 $\mu \mathrm{m}$ in length were briefly taken every 2-5 min for at least $1 \mathrm{hr}$ and collected as time series. Control experiments were performed by placing a slice culture under continuous perfusion and monitoring varicosity morphology for periods of $1-2 \mathrm{hr}$. The imaging time remained short (a few seconds every 2-5 min), and, usually, no signs of phototoxicity were detected. Occasionally, swellings could be observed, particularly after anoxia/hypoglycemia and not necessarily related to the imaged region. All these experiments were systematically discarded. We also assessed in blind studies the density of varicosities on the axonal segments under analysis. Although varicosity density could vary from axon to axon, the mean values obtained in this study are comparable for all conditions $(12.1 \pm 0.3$, $13.0 \pm 0.9,12.8 \pm 1.1$, and $11.6 \pm 0.7$ varicosities $/ 100 \mu \mathrm{m}$ in the control
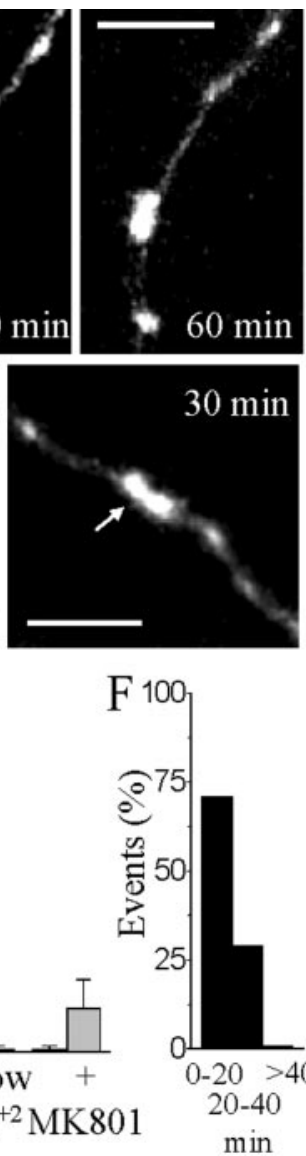

situation, anoxia/hypoglycemia, theta burst stimulation, and nitric oxide donor application, respectively). For the anoxia/hypoglycemia experiments, the morphology of varicosities was first monitored for $20 \mathrm{~min}$ to establish a baseline; anoxia/hypoglycemia was applied for $2 \mathrm{~min}$; the perfusion switched back to control, oxygenated medium; and imaging continued for at least 1 hr. Monitoring of synaptic responses during and after anoxia/hypoglycemia showed no evidence of epileptiform discharges. Also, these brief periods of anoxia/hypoglycemia (2 min) did not generally result in alterations of tissue morphology and cell preservation (Jourdain et al., 2002). The few cases in which swellings developed over time were discarded. For electrical stimulation experiments, the same protocol and criteria were used, except that labeled fibers were activated through an extracellular electrode placed in the vicinity of the labeled axon. The stimulation intensity was adjusted to produce a half-maximal field potential. After 20 min of baseline analysis, theta burst stimulation (TBS) was applied, and imaging continued for at least $1 \mathrm{hr}$. To assess the role of NMDA receptors in these mechanisms, anoxia/hypoglycemia and TBS experiments were repeated in the same way but with $40 \mu \mathrm{M}(+)$-5-methyl-10,11-dihydro5H-dibenzo[a,d]cyclohepten-5,10-imine maleate (MK801) added to the perfusion medium during the $20 \mathrm{~min}$ baseline period as well as during application of either anoxia/hypoglycemia or theta burst stimulation. The role of nitric oxide synthase inhibitors was investigated using the same protocol with $20 \mu \mathrm{M} \mathrm{N}$-omega-nitro-L-argininemethyl-ester (L-Name), a nonselective nitric oxide synthase inhibitor, or $50 \mu \mathrm{M}$ 3-bromo-7nitroindasole (3Br-7NI), a specific neuronal nitric oxide synthase inhibitor, added to the perfusion medium throughout the experiment. To test the effects of the nitric oxide donor, experiments were performed with $300 \mu \mathrm{M}$ 1,1-diethyl-2-hydroxy-2nitroso-hydrasine sodium (DEA/NO) applied for $10 \mathrm{~min}$ after a $20 \mathrm{~min}$ baseline, and varicosity morphology was assessed for another $60 \mathrm{~min}$ thereafter. Presynaptic filopodia-like structures were defined as dynamic protrusions of at least $0.5 \mu \mathrm{m}$ in length (measured from the varicosity or axon border to the tip of the filopodia) that appeared during the course of an experiment. No shape criteria were applied. Varicosity remodeling was defined as the transformation of a single varicosity into two smaller distinct and stable entities or the appearance during the course of the experiment of an axonal varicosity brighter than the axon shaft measuring at least $0.5 \mu \mathrm{m}$ and remaining stable throughout the observation period. All experiments were analyzed as time series by three different investigators, with the analysis being made blind by one of them.

Electron microscopy. All EM studies were performed on nonlabeled slice cultures. Slice cultures were fixed and processed as described (Toni et al., 1999; Jourdain et al., 2002). Briefly, cultures were fixed overnight at $4^{\circ} \mathrm{C}$ in $3 \%$ glutaraldehyde, rinsed in $0.1 \mathrm{M}$ phosphate buffer, $\mathrm{pH} 7.4$, and postfixed in a fresh solution of $1 \% \mathrm{OsO}_{4}$ with $1.5 \% \mathrm{~K}_{3} \mathrm{Cr}\left(\mathrm{C}_{2} \mathrm{O}_{4}\right)_{3}$ ( $\mathrm{Al}$ drich, Milwaukee, WI), $\mathrm{pH} 9.5$, for $2 \mathrm{hr}$. After a $5 \mathrm{~min}$ rinse in distilled water adjusted to $\mathrm{pH} 9.5$ with $\mathrm{KOH}$, the samples were dehydrated in ethanol and propylene oxide and embedded in Epon (Fluka, Buchs, Switzerland). For light microscopy, sections were stained with methylene blue. For serial electron microscopy, ribbons of up to 60 sections were cut in the middle portion of the apical arborization of CA1 pyramidal neurons (Ultratome Ultracut-E; Reichert-Jung) and collected on singleslotted Formvar-coated grids. Sections were stained for $20 \mathrm{~min}$ in $5 \%$ uranyl acetate and $30 \mathrm{sec}$ in lead citrate and analyzed on a Philips (Eind- 
hoven, The Netherlands) CM10 electron microscope at a magnification of $8900-28,500 \times$.

Axonal outgrowths or filopodia were defined as protrusions extending from a varicosity or an axon in a direction different from that of the axon and measuring a minimum of $0.5 \mu \mathrm{m}$ in length, calculated between the varicosity or axon external limit and the tip of the protrusion. To assess the involvement of filopodia-like structures in synapse formation, we performed threedimensional (3D) reconstructions of a total of 60 filopodia (17,20, and 23 at 10, 20, and 30 min after anoxia/hypoglycemia, respectively). Among the 17 shorter filopodia reconstructed $10 \mathrm{~min}$ after anoxia/hypoglycemia, we also included three cases in which the protrusion length was only between 0.4 and $0.5 \mu \mathrm{m}$ (see Fig. 4). Multiinnervated spines were defined by the presence of at least two distinct axonal terminals contacting the same spine. For stereological analyses, five or six serial sections per culture and three or four cultures per condition were examined, and synapses present in a volume of $218-1626 \mu \mathrm{m}^{3}$ were analyzed. The dissector procedure was performed as described previously (Geinisman et al., 1996). For the stereological analysis of filopodia, we randomly analyzed several (two to four) fields per culture (mean area per field, $637 \mu \mathrm{m}^{2}$ ) and, for each field, took overlapping photos (initial magnification, $8900 \times$ ) on each section from a set of four to six consecutive ultrathin serial sections. To recognize filopodia-like protrusions with certainty, they were followed through the whole set of sections. Then, for quantification, one of the sec-
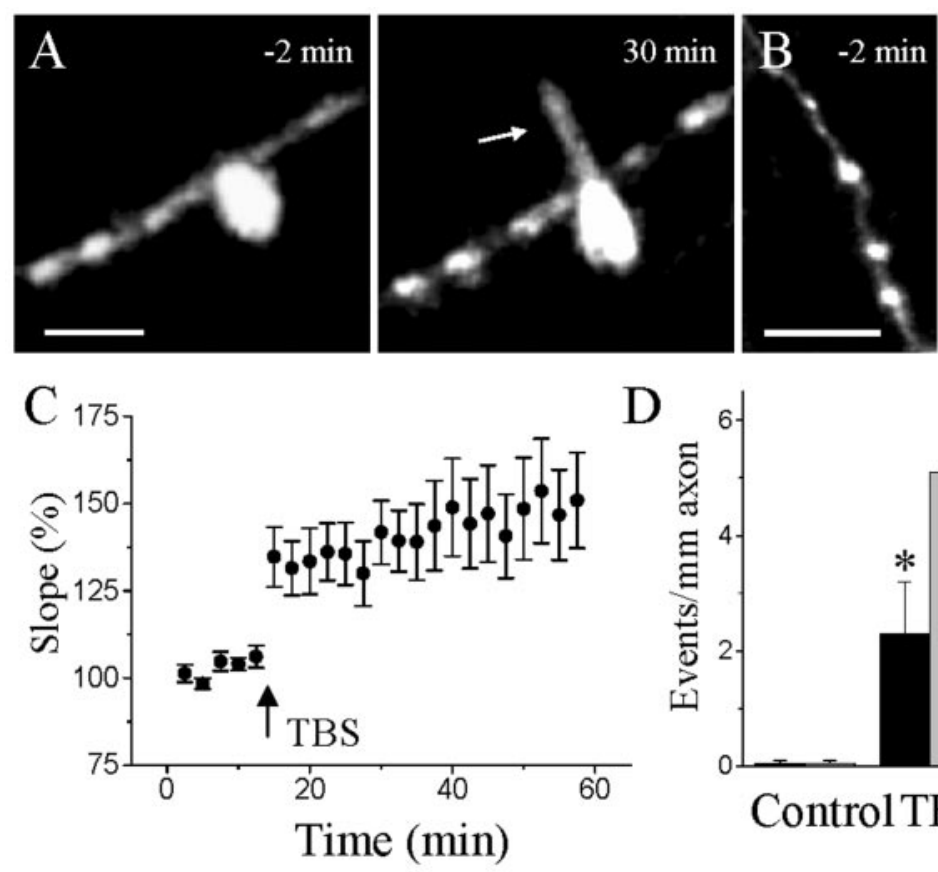

$\mathrm{D}$

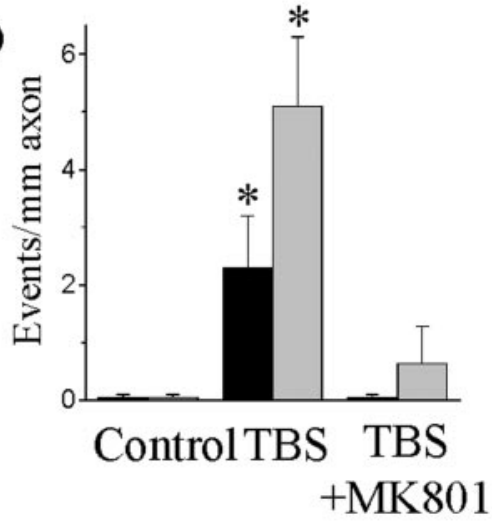

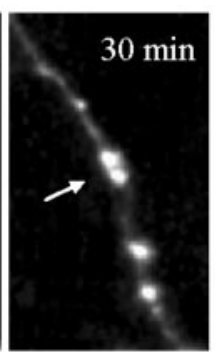

Figure 2. Confocal time-lapse imaging of presynaptic structural changes induced by TBS in Dil-labeled axons. $A$, Illustration of a presynaptic axonal outgrowth induced by TBS. Scale bar, $2 \mu \mathrm{m}$. B, Formation of a new varicosity observed after TBS. Scale bar, $5 \mu \mathrm{m}$. C, Changes in EPSP slope measured in the CA1 area on application of TBS through an electrode placed in the vicinity of labeled axons. Data are mean \pm SEM of 11 experiments. $D$, Statistical analysis of the number of axonal outgrowths (black columns) and varicosity remodeling (gray columns) detected under control conditions (no stimulation; $n=16$ ) and after theta burst stimulation applied in the absence $(n=17)$ or presence $(n=13)$ of $40 \mu \mathrm{M}$ MK801 ${ }^{*} p<0.05, t$ test).

tions was randomly chosen as a reference section, and the next one was chosen as a look-up one. We then counted the number of protrusions for which the contact zone between filopodia and varicosity or the axon of origin was visible on the reference section but not on the look-up one. For threedimensional analyses, axonal filopodia profiles were photographed serially at a magnification of at least $15,500 \times$ and $3 \mathrm{D}$-reconstructed using software developed by J. C. Fiala and K. M. Harris (Boston University, Boston, MA; http://synapses.mcg.edu/). Data are mean \pm SEM. Statistical analyses were performed using Student's $t$ test and Mann-Whitney $U$ test as mentioned.

\section{Results}

Previous work has shown that induction of long-term potentiation (LTP) as well as brief episodes of oxygen and glucose deprivation are associated with structural plasticity and a process of dendritic filopodia growth and formation of new spines (Engert and Bonhoeffer, 1999; Maletic-Savatic et al., 1999; Toni et al., 1999; Jourdain et al., 2002). Using repeated confocal imaging of DiI-labeled axons, here we investigated whether these conditions also involved presynaptic morphological changes. Under control conditions, analysis of presynaptic boutons over periods of at least 60 min revealed no detectable modifications of their morphology $(n=16 ; 1.98 \mathrm{~mm}$ analyzed; no spontaneous formation of filopodia-like structures, new varicosities, or varicosity remodeling). Axonal varicosities therefore appeared as stable structures in the absence of external manipulations. However, application of anoxia/hypoglycemia for 2 min was followed in 11 of 17 experiments by presynaptic modifications occurring within the next 60 min. We reported in the previous work that short anoxia/hypoglycemia resulted in NMDA receptor-dependent potentiation of synaptic transmission and structural remodeling of dendritic spines (Jourdain et al., 2002). The changes in EPSC evoked by short ( $2 \mathrm{~min}$ ) anoxia/hypoglycemia are illustrated in Figure $1 D$. Figure 1 demonstrates that this condition is also associated with presynaptic changes and illustrates the case of axonal outgrowth from a varicosity shown as a function of time (Fig. $1 A$ ). The axonal outgrowth appeared $5 \mathrm{~min}$ after anoxia/hypoglycemia; it rapidly extended to reach a maximal size at $20 \mathrm{~min}$ and then progressively retracted to disappear after $60 \mathrm{~min}$. Figure $1 B$ shows another example of axonal outgrowth that resembled a filopodia and started to grow $12 \mathrm{~min}$ after anoxia/hypoglycemia. A second type of change that could be observed was a process of varicosity remodeling that led to images suggesting a reorganization of a varicosity into two entities (Fig. 1C). These images could represent simple movements of vesicle packages, but, because they were stable once they appeared, they could also suggest the formation of a new varicosity or new release site. Although we could not elucidate the mechanisms underlying these images, we nevertheless assessed them because they appeared to depend on activity. Results were quantified by expressing for each experiment the number of events (axonal outgrowth and varicosity changes) detected per millimeter length of axon. In 17 experiments, analysis of axonal segments measuring a total of $1.89 \mathrm{~mm}$ revealed seven cases of growth of filopodia-like structures as well as seven cases of varicosity remodeling. When expressed in each experiment as the number of events per unit length of axonal segment, the increase was significant for filopodia and cases of varicosity remodeling (Fig. $1 E$; $p<0.05$, $t$ test; $p<0.05$, MannWhitney $U$ test).

To verify the specificity of this effect, we then performed the same experiments but in the presence of a medium containing low calcium $(0.1 \mathrm{~mm})$ and high magnesium $(10 \mathrm{~mm})$ to prevent synaptic activation. As shown in Figure $1 E$, the results of 12 experiments ( $1.55 \mathrm{~mm}$ of axonal segments analyzed) indicated that this medium completely prevented anoxia/hypoglycemiainduced morphological changes. Surprisingly, the same results were also obtained when repeating these experiments but in the presence of $40 \mu \mathrm{M}$ MK801, an NMDA receptor antagonist. In 16 

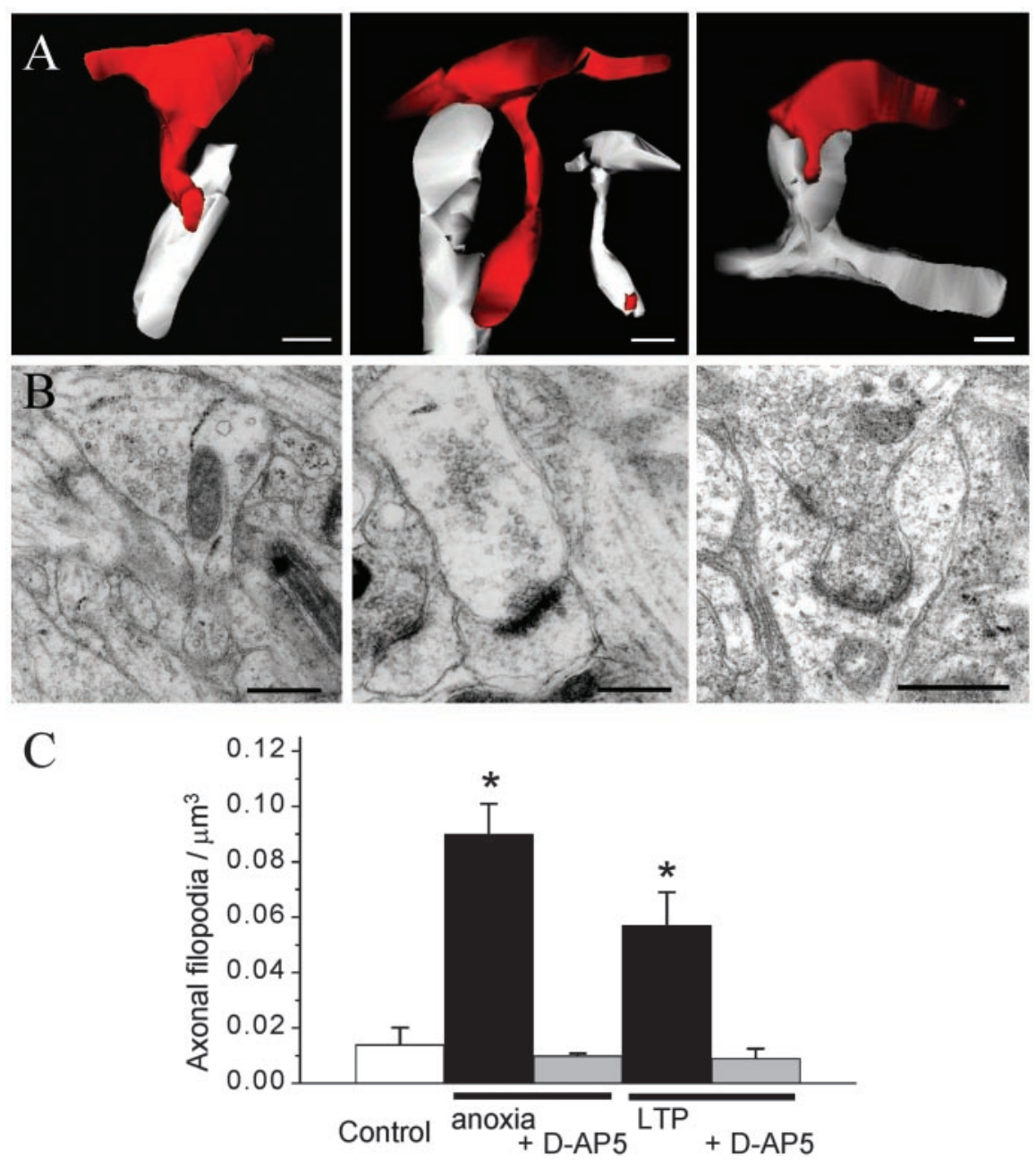

Figure 3. Electron microscopic analysis of presynaptic filopodia-like structures. $A$, Three examples of 3D-reconstructed presynaptic filopodia-like structures observed $20 \mathrm{~min}$ after anoxia/hypoglycemia (red). The examples on the left and right are also illustrated in $B$ (left, right) on a single section. The filopodia-like structure on the left was not contacting any PSD. The example illustrated in the middle contacted a dendritic shaft (inset, location of the PSD). The axonal outgrowth illustrated on the right was growing inside a postsynaptic spine and was associated with the formation of multiple PSDs ( $B$, right panel). $B$, Single-section illustrations of axonal filopodia-like structures obtained from tissue fixed $20 \mathrm{~min}$ after anoxia/hypoglycemia and showing the presence of synaptic vesicles in the presynaptic protrusions and different examples of contacts with membranes exhibiting either no PSD (left), a single PSD (middle), or multiple PSDs (right). Scale bars, $0.5 \mu \mathrm{m}$. C, Stereological analysis of the density of axonal outgrowths per cubic micrometer observed $20 \mathrm{~min}$ after anoxia/hypoglycemia or $30 \mathrm{~min}$ after TBS, in the presence or absence of the NMDA receptor antagonist D-AP-5 $\left({ }^{*} p<0.05, t\right.$ test).

experiments in which MK801 was applied during anoxia/hypoglycemia, no cases of axonal outgrowth and only two cases of varicosity remodeling were observed $(1.72 \mathrm{~mm}$ analyzed). When expressed as the number of events per millimeter length of axonal segments, these values were not different from those in the control situation and significantly decreased when compared with the anoxia/hypoglycemia condition (Fig. 1E; $p<0.05, t$ test). Figure $1 F$ shows in addition that these mechanisms of presynaptic remodeling were fairly rapid because most events were triggered during the first $20 \mathrm{~min}$ after anoxia/hypoglycemia (mean onset time, $12.5 \pm 1.8 \mathrm{~min}$, filopodia and varicosity remodeling). As illustrated in Figure 1 $\mathrm{A}$, axonal outgrowth usually occurred rapidly to reach a maximal size within 5-10 min after onset before tending to retract over the next $20-30 \mathrm{~min}$. In seven cases in which axonal outgrowths could be followed for $40-60 \mathrm{~min}$, two were seen to completely retract and disappear, whereas five were still present at the end of the experiment. In contrast, cases of varicosity remodel- ing, once induced, were generally stable for the time of observation (30-60 min).

We then performed experiments in which we electrically stimulated presynaptic endings through an extracellular electrode placed in the vicinity of the DiIlabeled axon. Stimulation pulses were delivered to evoke an EPSP recorded within the same area, and the stimulation intensity was adjusted to evoke halfmaximal responses. Monitoring of synaptic responses in 17 experiments showed that this protocol induced LTP (Fig. 2C). Confocal imaging of DiI-labeled axons revealed morphological changes in 12 of 17 experiments (1.98 $\mathrm{mm}$ of axonal segments analyzed). These also consisted of growth of filopodia-like protrusions ( $n=5$; Fig. $2 A)$ and cases of varicosity remodeling $(n=10$; Fig. $2 B)$. Their mean onset time after stimulation was $12.7 \pm 2.1 \mathrm{~min}$, similar to that observed with anoxia/hypoglycemia. When expressed as the number of events per unit length of axon per experiment, the increases were significant (Fig. $2 D ; p<0.05, t$ test). In control experiments in which low-frequency stimulation rather than a high-frequency train was applied, no morphological changes took place $(n=4)$. Furthermore, experiments performed in the same way, with the same stimulation protocol but in the presence of MK801 (40 $\mu \mathrm{M} ; n=13 ; 1.55 \mathrm{~mm}$ analyzed) showed that synaptic potentiation and morphological remodeling were prevented (Fig. $2 D ; p<0.05, t$ test). Thus, direct synaptic activation through theta burst stimulation was required to trigger these rapid presynaptic structural changes.

To investigate whether these presynaptic changes lead to the formation of new synaptic contacts, we then used electron microscopy, stereology, and 3D reconstruction. Consistent with confocal imaging data, stereological analyses revealed a marked increase in the density of images of presynaptic filopodia-like protrusions after both anoxia/hypoglycemia and LTP induction. Different examples of axonal outgrowths observed in these experiments are illustrated in Figure 3, $A$ and $B$. The left and right panels show the $3 \mathrm{D}$ reconstruction and a single-section illustration of the same axonal protrusion. The filopodia-like structure in the left panel was in close apposition with a dendritic shaft but without making a synaptic contact. The cases illustrated in the middle part represent filopodia-like structures that were in contact with postsynaptic membranes exhibiting a PSD. In the case illustrated in the right panels, the axonal protrusion has grown from the presynaptic varicosity inside the postsynaptic spine. In this example, the filopodia-like structure was making contact with multiple PSDs on the spine membrane. To quantify these changes, stereological analyses were performed using the dissector method in four control slice cultures, four slice cultures fixed $20 \mathrm{~min}$ after anoxia/hypoglycemia, and three slice cultures fixed $30 \mathrm{~min}$ after LTP induction. As illustrated in Figure 3, these 
experiments showed a significant increase in the density of filopodia-like structures per volume unit (control, $0.014 \pm 0.006 /$ $\mu \mathrm{m}^{3}$; anoxia/hypoglycemia, $0.090 \pm$ $0.011 / \mu \mathrm{m}^{3}$; LTP, $0.057 \pm 0.012 / \mu \mathrm{m}^{3} ; 271$, 269 , and $228 \mu \mathrm{m}^{3}$ analyzed; $\left.p<0.01\right)$. Interestingly, as for confocal imaging experiments, this increase was completely prevented when anoxia/hypoglycemia or LTP stimulation was applied in the presence of $100 \mu \mathrm{M}$ D-AP-5, a competitive NMDA receptor antagonist $(0.010 \pm 0.001$ and $0.007 \pm 0.003 / \mu \mathrm{m}^{3}$, respectively; $n=4$ and 3; 296 and $218 \mu \mathrm{m}^{3}$ analyzed).

To better study the progression and targets of these presynaptic filopodia-like structures, we then proceeded to $3 \mathrm{D}$ reconstruction of 17,23 , and 20 axonal outgrowths analyzed in three or four slices fixed 10, 20, and $30 \mathrm{~min}$ after anoxia/hypoglycemia, respectively. Figure $4 A$ illustrates examples of representative filopodia-like protrusions observed at these different time points. At 10 min after anoxia/hypoglycemia, axonal outgrowths were usually small, although most of them $(83 \%)$ already contained synaptic vesicles. In half of the cases (47\%), axonal outgrowths were not in contact with any postsynaptic target, and when this occurred, it was usually a dendritic shaft (Fig. $4 D)$. In only $18 \%$ of cases did the target exhibit a PSD. In contrast, axonal outgrowths seen 20 and $30 \mathrm{~min}$ after anoxia/ hypoglycemia were significantly longer and more mature (Fig. $4 A, B$ ). At $30 \mathrm{~min}$, they tended to retract (Fig. $4 B$ ), a phenomenon also observed with confocal imaging (Fig. $1 A$ ). Interestingly, a great proportion of them were now seen to contact a postsynaptic structure exhibiting a PSD (from $18 \%$ at $10 \mathrm{~min}$ to 78 and $90 \%$ at 20 and $30 \mathrm{~min}$, respectively; $p<0.05, t$ test; Fig. $4 C$ ). In addition, although the postsynaptic targets were often dendrites at 10 and $20 \mathrm{~min}$ (47 and $44 \%$ of cases, respectively), they had become mostly spines at $30 \mathrm{~min}(90 \%)$. Most of these spines (27 of 28 cases) were thin spines, and only one was a mushroom type of spine. Between 5 and $30 \mathrm{~min}$ after anoxia/hypoglycemia, the proportion of axonal filopodia-like structures contacting dendritic spines progressively increased from 6 to $90 \%$ (Fig. $4 D ; p<0.05, t$ test). At 30 $\mathrm{min}$, there were even a few cases in which the axonal outgrowths contacted two individual spines (Fig. $4 A$, right panel).

Additional evidence that axonal filopodia could lead to the formation of new synaptic contacts was the observation that, in some cases, filopodia-like structures contacted spines that were already innervated by another presynaptic terminal. This resulted in the formation of atypical synapses in which a spine had two distinct PSDs contacted each by a presynaptic terminal (Fig. 5A), a situation also found during development (Fiala et al., 1998). Using serial sectioning and stereological analyses, we assessed the number of these multi-innervated spines under control conditions and after anoxia/hypoglycemia or LTP induction. Their number increased significantly under both experimental conditions $(0.115 \pm 0.009$ and $0.103 \pm 0.008$ vs $0.030 \pm 0.006$ multiinnervated spines $/ \mu \mathrm{m}^{3}$ in anoxia/hypoglycemia, LTP, and control conditions, respectively; $n=4,3$, and 4 slice cultures; 1626,
1008, and $701 \mu \mathrm{m}^{3}$ analyzed; $p<0.01, t$ test; Fig. $5 B$ ), and the effect was prevented by pretreatment with $100 \mu \mathrm{M}$ D-AP-5 (Fig. $5 B) .3 \mathrm{D}$ reconstruction of 28 multi-innervated spines indicated that in all cases, synaptic vesicles were present in both presynaptic boutons, and in $82 \%$ of cases, multiple vesicles were docked to the membranes facing the two PSDs. Thus, these synapses had morphological features of functional contacts.

A surprising feature of this presynaptic structural plasticity was its dependence on activation of NMDA receptors. Antagonists of NMDA receptors prevented both the theta burst stimulation and anoxia/hypoglycemia-induced changes (Figs. 1E, 2D), a result further confirmed by stereological analyses (Figs. $3 C, 5 B$ ). In view of the postsynaptic location of NMDA receptors, we then investigated whether production and release of a retrograde messenger could eventually be involved. Among possible candidates, nitric oxide was of particular interest because it is an important modulator of excitatory synaptic transmission (Bon and Garthwaite, 2002). To test this possibility, we reproduced these experiments but in the presence of the inhibitor of nitric oxide synthase L-Name. In 15 experiments in which anoxia/hypoglycemia was applied in the presence of $20 \mu \mathrm{M} \mathrm{L}$-Name $(1.85 \mathrm{~mm}$ of axonal segments analyzed), no changes in presynaptic morphology could be detected, a result much different from that produced by anoxia/hypoglycemia alone (Fig. $6 B ; p<0.05, t$ test). Figure $6 A$ illustrates one such experiment. To examine this further, we also performed 14 experiments in which theta burst stimulation was applied in the presence of L-Name (1.68 $\mathrm{mm}$ analyzed). In these 14 experiments, only one case of varicosity remodeling could be 

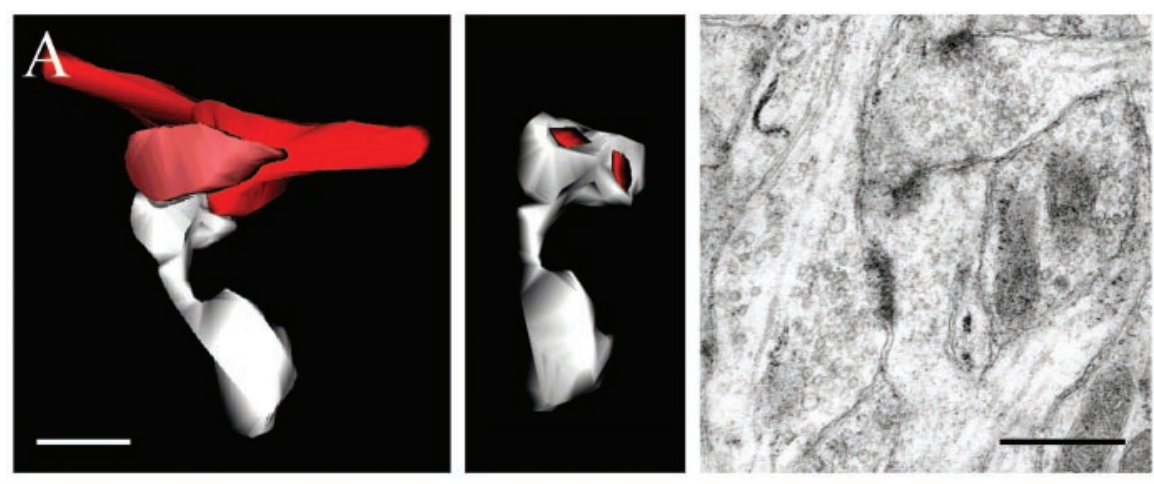

B
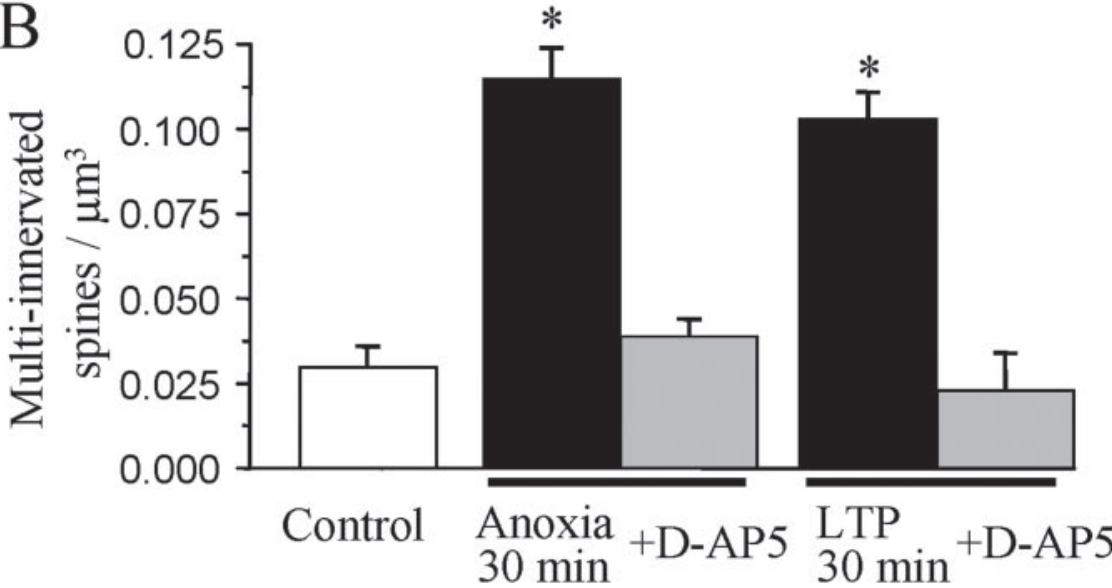

Figure 5. Contribution of axonal remodeling to the formation of multi-innervated spines. $A$, Three-dimensional reconstruction of a multi-innervated spine (left) contacted by two distinct terminals. Middle, Same spine illustrating the presence of two distinct PSDs. Right, Single section illustrating a multi-innervated spine having PSDs with two distinct boutons filled with vesicles. Scale bars, $0.5 \mu \mathrm{m}$. B, Stereological analysis of the density of multi-innervated spines per cubic micrometer in the conditions tested $\left({ }^{*} p<0.05, t\right.$ test $)$.

observed, whereas theta burst stimulation alone has resulted in 17 experiments in the growth of five filopodia-like structures and 10 cases of varicosity changes, a result significantly different from that obtained with L-Name (Fig. $6 B ; p<0.05, t$ test). In additional experiments, we also tested the effect of $3 \mathrm{Br}-7 \mathrm{NI}(50 \mu \mathrm{M})$, another selective inhibitor of neuronal nitric oxide synthase. The results of five experiments again revealed that no structural changes occurred under this condition (data not shown). Taken together, these data indicated that blockade of nitric oxide production markedly prevented the structural presynaptic changes induced by anoxia/hypoglycemia and by theta burst stimulation.

If nitric oxide is a retrograde messenger responsible for the growth of filopodia and remodeling of varicosities, then adding nitric oxide to the tissue should be able to reproduce these effects. We investigated this using the nitric oxide donor DEA/NO. Fifteen experiments were performed by incubating slice cultures with $300 \mu \mathrm{m}$ DEA/NO for $10 \mathrm{~min}$. The effect of DEA/NO could be easily monitored electrophysiologically because it produced a transient depression followed by a rebound potentiation, as recently reported by Bon and Garthwaite (2002) (Fig. 7C). When analyzing the morphology of presynaptic varicosities, we found that presynaptic morphological changes took place in 9 of 15 experiments, with 5 cases of axonal outgrowths and 10 cases of varicosity remodeling ( $1.8 \mathrm{~mm}$ of axonal segments analyzed; Fig. $7 A$ ). When compared with a control situation in which no drug was added, the changes were statistically significant (Fig. $7 B ; p<$ $0.05, t$ test). Thus, a nitric oxide donor was able to reproduce the presynaptic remodeling triggered by anoxia/hypoglycemia and theta burst stimulation.

\section{Discussion}

The results of this study show that induction of LTP and application of a short period of anoxia/hypoglycemia can trigger a presynaptic morphological plasticity characterized by the growth of presynaptic filopodia-like structures and remodeling of varicosities. These observations are consistent with recent evidence demonstrating activity-dependent remodeling of presynaptic actin and formation of new puncta in Aplysia neurons, dissociated cell cultures, and organotypic slice cultures (Hatada et al., 2000; Chang and De Camilli, 2001; Colicos et al., 2002; De Paola et al., 2003; Tashiro et al., 2003). Our study demonstrates that these mechanisms also contribute to activity-dependent plasticity in the hippocampus and that they participate, at least in the short term, in synaptogenesis and probably spine formation.

Evidence for presynaptic remodeling was obtained both by confocal imaging of DiI-labeled axonal segments and stereological analyses of serial sections and 3D reconstruction of presynaptic structures. The two approaches gave very consistent and comparable results with regard to both the time course of axonal filopodialike structures and their number. Axonal outgrowths started to appear 5-15 min after stimulation; they reached a maximum size after $\sim 20 \mathrm{~min}$; their maximum length was in both cases $\sim 1 \mu \mathrm{m}$; and their numbers reached similar values $(3.6 \pm$ $1.3 \%$ of examined varicosities with confocal imaging and $5.2 \pm$ $0.7 \%$ of total synapses with stereology). Also, analysis of $3 \mathrm{D}$ reconstructed filopodia-like structures clearly shows that they are able to form fully morphologically mature synaptic contacts. The surprising observation is the rapidity of the phenomenon. The presynaptic changes were triggered at approximately the same time as dendritic filopodia (Maletic-Savatic et al., 1999; Jourdain et al., 2002). Electron microscopic analyses confirmed this result, and 3D-reconstruction experiments further indicated that axonal filopodia-like structures observed after 20-30 min are for most of them in contact with a PSD. At that time, the contacts formed by axonal outgrowths exhibited all morphological characteristics of mature and functional synapses; the presynaptic protrusions were filled with vesicles, some of which were docked to the active zone membrane facing the PSD. A simple interpretation of these results is thus that synaptic activity triggered the growth of presynaptic filopodia-like structures that subsequently induced the formation of a PSD on a target cell. This timing is consistent with that required for the migration and formation of new active zones (Kraszewski et al., 1995; Ahmari et al., 2000); it also corresponds to the time necessary for dendritic filopodia to grow, retract, and then stabilize as new spine synapses (Dailey and Smith, 1996; Ziv and Smith, 1996; Fiala et al., 1998; MaleticSavatic et al., 1999; Jourdain et al., 2002). Finally, it takes place within a time frame that is compatible with that required for the formation of a new PSD, as revealed by analyses of the dynamics of PSD proteins (Marrs et al., 2001). Taken together, these data suggest that a presynaptic growth process, such as the appearance 

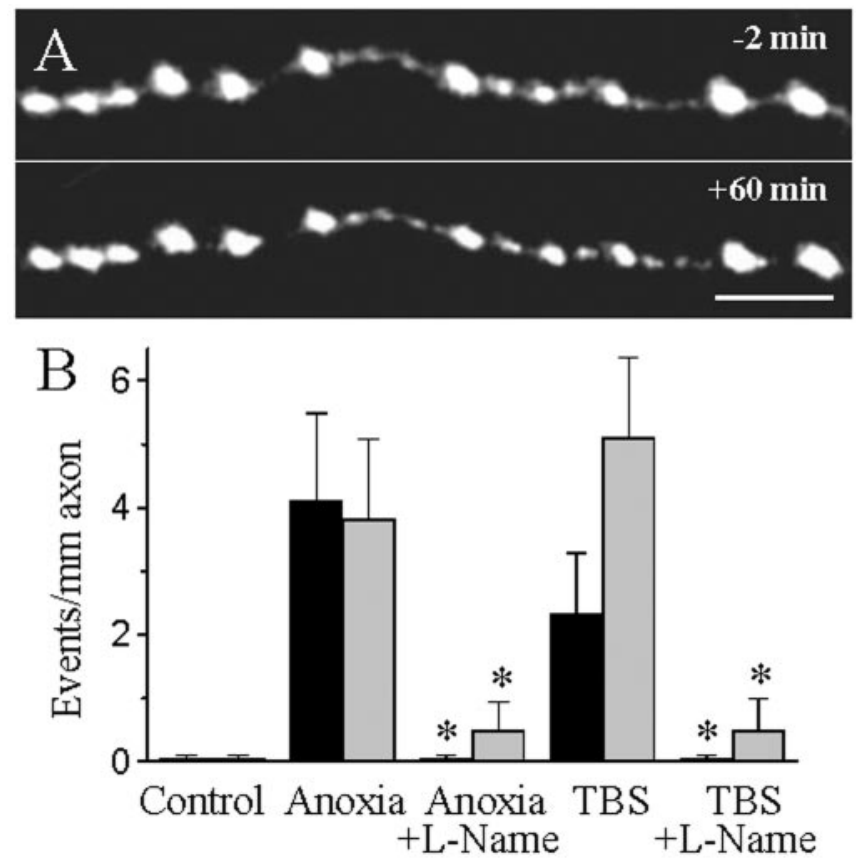

Figure 6. Blockade by an inhibitor of nitric oxide synthase of presynaptic morphological changes induced by anoxia/hypoglycemia and theta burst stimulation. A, One of 15 experiments in which application of a short anoxia/hypoglycemia in the presence of $20 \mu \mathrm{m} \mathrm{L}$-Name failed to trigger changes in the morphology of varicosities. Scale bar, $5 \mu \mathrm{m}$. B, Quantitative analyses showing that the growth of presynaptic axonal protrusions (black columns) and cases of varicosity remodeling (gray columns) expressed as events per millimeter of axon were blocked when anoxia/hypoglycemia and theta burst stimulation were applied in the presence of $20 \mu \mathrm{m}$ L-Name ( $n=15$ and 14 , respectively; ${ }^{*} p<0.05, t$ test).

of an axonal outgrowth, can lead to formation of a morphologically mature synapse within $\sim 0.5 \mathrm{hr}$.

This presynaptic structural plasticity was clearly under the control of synaptic activity and thus probably contributed not only to developmental plasticity but also to activity-dependent structural remodeling. The presynaptic changes observed by confocal imaging were both calcium- and NMDA-receptor dependent, and when induced by electrical stimulation, they required high-frequency stimulation. These characteristics are thus very similar to those necessary to induce LTP (Malenka and Nicoll, 1999). The dependence on NMDA receptor activation, observed both with confocal and electron microscopic analyses, was particularly surprising, although consistent with a result also obtained in dissociated cell cultures (Colicos et al., 2002). It must be noted, however, that other studies in dissociated cell cultures and organotypic slices cultures instead reported AMPA and kainate receptor dependency (Chang and De Camilli, 2001; De Paola et al., 2003; Tashiro et al., 2003). Importantly, recent studies by De Paola et al. (2003) and Tashiro et al. (2003) were performed in the CA3 and not CA1 area, thus raising the possibility that there could be region-specific differences, as for LTP properties. Tashiro et al. (2003) further reported different developmental sensitivities, suggesting that the modulation could be complex. Our results do not exclude a role for AMPA receptors in these mechanisms or a presynaptic action of released glutamate. The effect, however, should be mediated by presynaptic NMDA receptors because the antagonists used blocked presynaptic remodeling without affecting postsynaptic AMPA-mediated responses. Because there is yet no clear evidence for a presynaptic location of NMDA receptors in CA1, we instead investigated the possibility that this presynaptic structural plasticity depended on activation of postsynaptic NMDA receptors and required the involvement
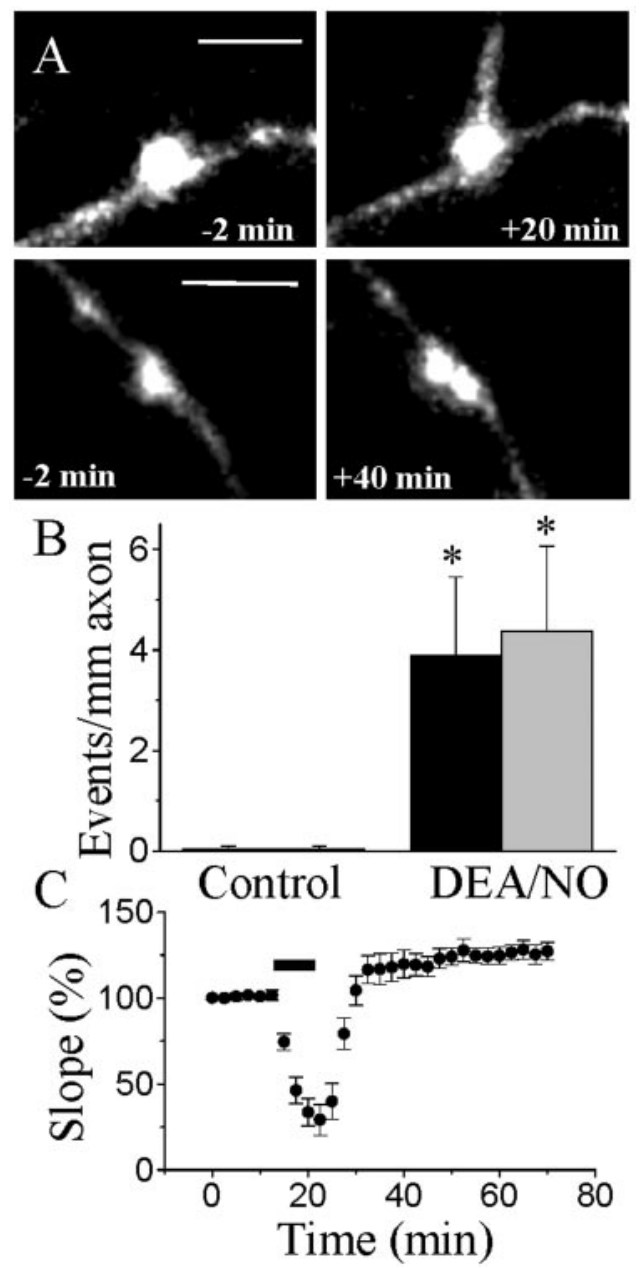

Figure 7. A nitric oxide donor reproduces the presynaptic morphological plasticity induced by anoxia/hypoglycemia and theta burst stimulation. $A, 0$ ne case of presynaptic axonal outgrowth (top) and one case of varicosity formation (bottom) observed after a 10 min application of $300 \mu \mathrm{m}$ DEA/NO, a nitric oxide donor. Scale bars, $2 \mu \mathrm{m}$. $B$, Number of axonal outgrowths (black columns) and varicosity remodeling (gray columns) per millimeter of axon observed within 60 min under control conditions (no drug applied) and after application of $300 \mu \mathrm{m}$ DEA/NO for $10 \mathrm{~min}$ ( $n=15 ;{ }^{*} p<0.05, t$ test). C, Changes in EPSP slope induced by application of the nitric oxide donor DEA/NO (300 $\mu \mathrm{m})$ for $10 \mathrm{~min}$ (bar). Data are mean \pm SEM of nine experiments.

of a retrograde messenger. The fact that inhibitors of nitric oxide synthase prevented this presynaptic structural plasticity, and a nitric oxide donor reproduced it suggests that nitric oxide could be this retrograde messenger. Such a role of nitric oxide in modulating structural plasticity is not completely unexpected. Nitric oxide synthase is present in spines in the hippocampus (Burette et al., 2002), and work from many laboratories has provided evidence for involvement of nitric oxide in synaptic physiology and modulation of presynaptic properties (Hawkins et al., 1998; Bon and Garthwaite, 2002). A possible role of nitric oxide in LTP mechanisms has even been proposed, although this issue remains controversial (Hawkins et al., 1998; Malenka and Nicoll, 1999). Nitric oxide also affects growth processes by interfering with the polymerization of actin filaments (Hindley et al., 1997; Ernst et al., 2002). Taken together, these results are consistent with a model in which activation of NMDA receptors and calcium influx in spines could trigger the release of nitric oxide, which in turn would diffuse locally and induce a growth process in presynaptic boutons and axons. This growth process, coordinated with postsynaptic structural plasticity, could rapidly lead to the forma- 
tion of new synaptic contacts. Interestingly, the growth of presynaptic filopodia-like structures could be directly implicated in the appearance of some of the new spines described under the same conditions (Engert and Bonhoeffer, 1999; Jourdain et al., 2002). As the 3D-reconstruction analyses show, filopodia were found to initially contact mainly dendritic shafts and then later, almost exclusively, spines. This sequence thus strongly suggests that axonal outgrowths start to make shaft synapses that are later transformed into spine synapses. The timing of the appearance of the new spines revealed by confocal imaging perfectly coincides with the moment when the postsynaptic target of axonal protrusions switches from dendrites to spines. According to this model, postsynaptic NMDA receptor-dependent mechanisms could induce structural synaptic remodeling not only by stimulating postsynaptic processes such as the growth of dendritic filopodia or the formation of new spines (Ziv and Smith, 1996; Fiala et al., 1998; Engert and Bonhoeffer, 1999; Maletic-Savatic et al., 1999) but also by triggering a presynaptic morphological plasticity that would play an active role in synaptogenesis and formation of new spine synapses. Our observation of multi-innervated spines in which presynaptic outgrowths are involved and evidence showing that prolonged sensory stimulation also leads to the formation of multi-innervated spines (Knott et al., 2002) further support this interpretation. It remains, however, to determine what could be the long-term stability of these new synapses. Two recent studies performed in vivo suggest that newly formed spines can be stable over prolonged periods (Grutzendler et al., 2002; Trachtenberg et al., 2002). Similarly, analyses of the turnover of presynaptic varicosities suggest that a significant fraction of them are likely to be very stable (De Paola et al., 2003). The advantage of the model presented here is that it makes predictions about the mechanisms involved that are testable and could provide additional insights into the complex and probably diverse interactions likely to take place between presynaptic and postsynaptic structures in the mechanisms of activity-dependent synaptogenesis.

\section{References}

Ahmari SE, Buchanan J, Smith SJ (2000) Assembly of presynaptic active zones from cytoplasmic transport packets. Nat Neurosci 3:445-451.

Bon CL, Garthwaite J (2002) Exogenous nitric oxide causes potentiation of hippocampal synaptic transmission during low-frequency stimulation via the endogenous nitric oxide-cGMP pathway. Eur J Neurosci 14:585-594.

Buchs PA, Muller D (1996) Induction of long-term potentiation is associated with major ultrastructural changes of activated synapses. Proc Natl Acad Sci USA 93:8040-8045.

Burette A, Zabel U, Weinberg RJ, Schmidt HH, Valtschanoff JG (2002) Synaptic localization of nitric oxide synthase and soluble guanylyl cyclase in the hippocampus. J Neurosci 22:8961-8970.

Chang S, De Camilli P (2001) Glutamate regulates actin-based motility in axonal filopodia. Nat Neurosci 4:787-793.

Colicos MA, Collins BE, Sailor MJ, Goda Y (2002) Remodeling of synaptic actin induced by photoconductive stimulation. Cell 107:605-616.

Dailey ME, Smith SJ (1996) The dynamics of dendritic structure in developing hippocampal slices. J Neurosci 16:2983-2994.

De Paola V, Arber S, Caroni P (2003) AMPA receptors regulate dynamic equilibrium of presynaptic terminals in mature hippocampal networks. Nat Neurosci 6:491-500.

Engert F, Bonhoeffer T (1999) Dendritic spine changes associated with hippocampal long-term synaptic plasticity. Nature 399:66-70.

Ernst AF, Gallo G, Letourneau PC, McLoon SC (2002) Stabilization of growing retinal axons by the combined signaling of nitric oxide and brain-derived neurotrophic factor. J Neurosci 20:1458-1469.

Fiala JC, Feinberg M, Popov V, Harris KM (1998) Synaptogenesis via dendritic filopodia in developing hippocampal area CA1. J Neurosci 18:8900-8911.

Fischer M, Kaech S, Knutti D, Matus A (1998) Rapid actin-based plasticity in dendritic spines. Neuron 20:847-854.

Geinisman Y (1993) Perforated axospinous synapses with multiple, com- pletely partitioned transmission zones: probable structural intermediates in synaptic plasticity. Hippocampus 3:417-434.

Geinisman Y, Gundersen HJ, van der Zee E, West MJ (1996) Unbiased stereological estimation of the total number of synapses in a brain region. J Neurocytol 25:805-819.

Goldin M, Segal M, Avignone E (2001) Functional plasticity triggers formation and pruning of dendritic spines in cultured hippocampal networks. J Neurosci 21:186-193.

Grutzendler J, Kasthuri N, Gan WB (2002) Long-term dendritic spine stability in the adult cortex. Nature 420:812-816.

Hatada Y, Wu F, Sun ZY, Schacher S, Goldberg DJ (2000) Presynaptic morphological changes associated with long-term synaptic facilitation are triggered by actin polymerization at preexisting varicosities. J Neurosci 20:RC82(1-5).

Hawkins RD, Son H, Arancio O (1998) Nitric oxide as a retrograde messenger during long-term potentiation in hippocampus. Prog Brain Res 118:155-172.

Hindley S, Juurlink BH, Gysbers JW, Middlemiss PJ, Herman MA, Rathbone MP (1997) Nitric oxide donors enhance neurotrophin-induced neurite outgrowth through a cGMP-dependent mechanism. J Neurosci Res 47:427-439.

Jourdain P, Nikonenko I, Alberi S, Muller D (2002) Remodeling of hippocampal synaptic networks by a brief anoxia-hypoglycemia. J Neurosci 22:3108-3116.

Kirov SA, Harris KM (1999) Dendrites are more spiny on mature hippocampal neurons when synapses are inactivated. Nat Neurosci 2:878-883.

Knott GW, Quairiaux C, Genoud C, Welker E (2002) Formation of dendritic spines with GABAergic synapses induced by whisker stimulation in adult mice. Neuron 34:265-273.

Korkotian E, Segal M (1999) Release of calcium from stores alters the morphology of dendritic spines in cultured hippocampal neurons. Proc Natl Acad Sci USA 96:12068-12072.

Korkotian E, Segal M (2001) Spike-associated fast contraction of dendritic spines in cultured hippocampal neurons. Neuron 30:751-758.

Kraszewski K, Mundigl O, Daniell L, Verderio C, Matteoli M, De Camilli P (1995) Synaptic vesicle dynamics in living cultured hippocampal neurons visualized with Cy3-conjugated antibodies directed against the luminal domain of synaptotagmin. J Neurosci 15:4328-4342.

Luscher C, Nicoll RA, Malenka RC, Muller D (2000) Synaptic plasticity and dynamic modulation of the postsynaptic membrane. Nat Neurosci 3:545-550

Ma L, Zablow L, Kandel ER, Siegelbaum SA (1999) Cyclic AMP induces functional presynaptic boutons in hippocampal CA3-CA1 neuronal cultures. Nat Neurosci 2:24-30.

Malenka RC, Nicoll RA (1999) Long-term potentiation: a decade of progress? Science 285:1870-1874.

Maletic-Savatic M, Malinow R, Svoboda K (1999) Rapid dendritic morphogenesis in CA1 hippocampal dendrites induced by synaptic activity. Science 283:1923-1927.

Marrs GS, Green SH, Dailey ME (2001) Rapid formation and remodeling of postsynaptic densities in developing dendrites. Nat Neurosci 4:1006-1013.

Segal I, Korkotian I, Murphy DD (2000) Dendritic spine formation and pruning: common cellular mechanisms? Trends Neurosci 23:53-57.

Star EN, Kwiatkowski DJ, Murthy VN (2002) Rapid turnover of actin in dendritic spines and its regulation by activity. Nat Neurosci 5:239-246.

Stoppini L, Buchs P-A, Muller D (1991) A simple method for organotypic cultures of nervous tissue. J Neurosci Methods 37:173-182.

Tashiro A, Dunaevsky A, Blazeski R, Mason CA, Yuste R (2003) Bidirectional regulation of hippocampal mossy fiber filopodial motility by kainate receptors: a two-step model of synaptogenesis. Neuron 38:773-784.

Trachtenberg JT, Chen BE, Knott GW, Feng G, Sanes JR, Welker E, Svoboda $\mathrm{K}$ (2002) Long-term in vivo imaging of experience-dependent synaptic plasticity in adult cortex. Nature 420:788-794.

Toni N, Buchs PA, Nikonenko I, Bron CR, Muller D (1999) LTP promotes formation of multiple spine synapses between a single axon terminal and a dendrite. Nature 402:421-425.

Toni N, Buchs PA, Nikonenko I, Povilaitite P, Parisi L, Muller D (2001) Remodeling of synaptic membranes after induction of long-term potentiation. J Neurosci 21:6245-6251.

Ziv NE, Smith SJ (1996) Evidence for a role of dendritic filopodia in synaptogenesis and spine formation. Neuron 17:91-102. 